RUNNING HEAD: COVID-19 AND STUDENT MENTAL HEALTH

The Impact of the COVID-19 Pandemic on College Student Mental Health: A Longitudinal

\title{
Examination of Risk and Protective Factors
}

\author{
Martha Zimmermann, MA $^{1}$ \\ University of Nevada, Reno \\ Casandra Bledsoe, BA \\ University of Nevada, Reno \\ Anthony Papa, $\mathrm{PhD}$ \\ University of Nevada, Reno
}

Declarations of interest: none.

University of Nevada Reno

Department of Psychology

1664 N. Virginia St., Reno, NV, 89557

United States

${ }^{1}$ Martha Zimmermann

University of Nevada Reno

Department of Psychology

1664 N. Virginia St., Reno, NV, 89557

United States

e-mail: mzimmermann@nevada.unr.edu 


\section{Highlights}

- A longitudinal study examined student distress during the 2019-2020 academic year

- College students experienced an increase in distress during the COVID-19 pandemic

- Pre-existing symptom severity predicted distress during the pandemic

- Cognitive and behavioral avoidance and internet use predicted distress

- Women and Latinx students reported more distress during the COVID-19 pandemic 


\begin{abstract}
The spread of the novel coronavirus has led to unprecedented changes in daily living. College students may face unique challenges associated with these changes. In the present study, college students $(N=205)$ completed a battery of questionnaires in April of 2020, after having completed similar measures 8, 5, and 2 months prior as part of a larger study. A repeated measures ANOVA suggested significantly greater depression and anxiety symptom severity during the pandemic than at any other time during the 2019-2020 academic year. Static and modifiable factors associated with psychological distress and controlling for pre-existing psychological distress were examined. Cognitive and behavioral avoidance was the most consistent predictor of psychological distress during the pandemic. Online social engagement and problematic Internet use also conferred greater risk. Women and Latinx participants were more likely to experience elevated distress during the pandemic, even when controlling for distress prior to the pandemic.
\end{abstract}

Keywords: COVID-19, college students, depression, anxiety, distress 


\section{The Impact of the COVID-19 Pandemic on College Student Mental Health: A Longitudinal Examination of Risk and Protective Factors}

\section{Introduction}

The novel coronavirus (SARS-CoV-2) has taken an enormous toll since its spread began in December of 2019 (Zhou et al., 2020). The virus and resulting acute respiratory syndrome (COVID-19) resulted in more than 1.7 million confirmed cases and over 100,000 deaths in the United States by the end of May 2020 according to data from the COVID-19 Dashboard by the Center for Systems Science and Engineering (CSSE) at Johns Hopkins University (Dong, Du, \& Gardner, 2020).

In addition to the effect of the virus on human health, social distancing measures and shelter-in-place orders haves led to unprecedented changes in daily life that likely have serious implications for mental health (Gruber et al., 2020). A review examining quarantine measures to prevent the spread of other pandemics (e.g., SARS in 2003, Ebola in 2014) found myriad psychological consequences of quarantine (Brooks et al., 2020). Accordingly, initial reports have suggested that COVID-19 pandemic may have already led to poorer mental health in the general public. A large cross-sectional study of Chinese adults a significant psychological impact of the virus, with the majority of participants $(75.2 \%)$ reporting worry about the health of family members, $16.5 \%$ reported moderate to severe depressive symptoms, and $28.8 \%$ reported moderate to severe anxiety symptom severity (Wang et al., 2020). Predictors of psychological distress in this sample included female gender, being a student, and physical health, while engaging in preventive measures (e.g., hand-washing, mask wearing) was associated with lower distress. A study conducted in Italy in mid-April of 2020 found that $38 \%$ of participants indicated mild to moderate psychological distress (Moccia et al., 2020). Female gender was 
again associated with greater distress. Results also suggested some trait-level individual characteristics associated with greater distress such as anxious temperament.

\subsection{College students}

While college students may be at lower risk for serious complications associated with COVID-19, they face significant disruption following school closures and social distancing measures. Increases in depression, anxiety, and irritability were frequently reported. It has been suggested that emerging adulthood (ages 18-24) may represent a developmental stage with unique challenges imposed by COVID-19 (Gruber et al., 2020). These challenges may include disrupted social roles as a result of missing important experiences, returning to living with parents, and financial difficulties. These factors may explain greater risk of distress among this population compared to their non-student counterparts (Wang et al., 2020).

Quarantine, and shelter-in-place measures, may of be particular relevance to mental health during pandemics. Specifically, past pandemics and the related quarantine have been shown to be associated with negative psychological effects, although factors that confer risk for distress are less well established (Brooks et al., 2020). Among college students in China, urban residence, family income instability, not living with family, and relative or acquaintance with COVID-19 were found to be associated with more severe anxiety during the COVID-19 pandemic (Cao et al., 2020). Less is known about modifiable risk factors, or those that could be reasonably altered by the individual (e.g., Cairns, Yap, Pilkington, \& Jorm, 2014). What's more, the majority of studies examining the psychological impact of pandemics are cross-sectional in nature, limiting the ability to examine both the magnitude of change in distress and prequarantine factors that confer risk. As such, the prospective examination of anxiety and depression following the COVID-19 spread is essential to understanding the scale of the 
psychological impact as well as risk and protective factors. The goal of the present study was to examine the impact of COVID-19 in a convenience sample of participants enrolled in a separate study at the beginning of the 2019-2020 academic school year.

\section{Method}

\subsection{Participants and Procedures}

Participants were college students at a large public university it the United States who enrolled in a study beginning in August 2019 examining the effects of a depression and anxiety prevention program. In the original study, students $(N=371)$ completed self-report measures assessing mental health status and health behavior on three occasions prior to disruptions that occurred as a result of the COVID-19 pandemic (August 2019, December 2019, February 2020). By the third data collection point (end of February 2020), 24 confirmed cases of COVID-19 were reported in the United States. A state of Emergency for the State of Nevada was declared on March $15^{\text {th }}$, and college students moved to remote instruction and operations on shortly afterwards. Students in residential living were instructed to relocate off campus at this time. All participants completing the six-month follow up $(N=278)$ were invited to participate in an additional follow up on April $3^{\text {rd }}, 2020$, of which 205 participated. By the final date of collection (April $20^{\text {th }}$ ), 779,700 confirmed cases were reported by the COVID-19 Dashboard by the Center for Systems Science and Engineering (CSSE) at Johns Hopkins University (Dong et al., 2020). Participants were a mean age of $18.4(S D=.99)$ and primarily $(76.2 \%)$ female. Just over half of participants identified as White (53.9\%), 18.4\% identified as Asian/Pacific Islander, 16.1\% Latinx, 4.4\% identified as Black or African American, and 6.8\% identified as other. Of the sample, $37.7 \%$ reported an income below $\$ 49,999,32.0 \%$ reported an income between $\$ 50,000$ and $\$ 99,9999$, and $29.9 \%$ reported an income over $\$ 100,000$. 


\subsection{Measures}

\subsubsection{Pre and Post Pandemic}

2.2.1.1 Depression severity was assessed using a 9-item questionnaire that identifies DSM symptoms for Major Depressive Disorder (PHQ-9; Kroenke, Spitzer, \& Williams, 2001; $\alpha$ $\left.=.90, .90^{1}\right)$. Scoring 10 or above on the PHQ-9 is associated with a sensitivity of of $88 \%$ and a specificity of $88 \%$ for Major Depressive Disorder.

2.2.1.2 Anxiety symptoms were measured using a commonly used 7-item scale which evaluates symptoms for Generalized Anxiety Disorder (GAD-7; Spitzer, Kroenke, Williams, \& Löwe, 2006; $\alpha=.93, .92)$. Scoring 10 or above on the GAD-7 is associated with a sensitivity of $89 \%$ and specificity of $82 \%$ for an anxiety disorder diagnosis.

2.2.1.3 Physical activity was measured using a single item ("How many times a week do you usually do 30 minutes of moderate physical activity or walking that increases your heart rate or makes you breath harder than normal? (for example, mowing the lawn, carrying light loads, bicycling at a regular pace, or playing doubles tennis)." Participants indicated, "none" "1-2 times/week", "3-4 times/week”, or "> 5 times/week" (Marshall, Smith, Bauman, \& Kaur, 2005).

2.2.1.4 Social Engagement was assessed using four items adapted from a scale assessing college student social contact (Kim, Wang, \& Oh, 2016; $\alpha=.88, .72$ ). While Cronbach's alpha was acceptable at T3, $(\alpha=.78)$, unsurprisingly it was not acceptable at T3 $(\alpha=.57)$ as the first two items ["Attended gatherings with friends or family", and "Attended organized events (e.g., school, work, other social group)]" were likely substantially impacted by COVID-19 and related disruptions and as such unrelated to the final two items ("Spent time with friends or family" and

\footnotetext{
${ }^{1}$ Cronbach's alpha represents value at T3 (February 2020) and T4 (April 2020).
} 
"Chatted with friends or family"). As such, only the second two items were used to examine inperson social contact.

2.2.1.5 Hours of sleep were assessed using the single item ("On the average, how many hours did you sleep each night during the past 4 weeks?’) from the Medical Outcomes Study Sleep measure (MOS; Hays, Martin, Sesti, \& Spritzer, 2005).

2.2.1.6 Dietary behavior was assessed using a 10-item assessment of healthy dietary habits (e.g., "Do you try to eat less sweets and pastries?"; Zazpe et al., 2011; $\alpha=.76, .80$ ). Scores range from 0 to 10 , with higher scores indicating a greater number of eating habits designated as healthy.

2.2.1.7 Perceived malleability of emotions was measured using a 4-item scale developed by Tamir and colleagues $(2007 ; \alpha=.79, .80)$. High scores represent a belief that one's emotions are malleable whereas low scores indicate the belief that one's emotions are fixed and unchangeable (Schroder, Dawood, Yalch, Donnellan, \& Moser, 2016; Tamir, 2007). Believing emotions to be malleable been found to be associated with less psychological distress in several studies (Ford, Lwi, Gentzler, Hankin, \& Mauss, 2018; Kneeland \& Dovidio, 2019; Tamir, 2007).

2.2.1.8 Cognitive and behavioral avoidance was measured using a 31-item scale known as the Cognitive-Behavioral Avoidance Scale (CBAS; Ottenbreit \& Dobson, 2004; $\alpha=.96, .96$ ). The scale covers four aspects of avoidance, including cognitive avoidance, behavioral avoidance, social and non-social avoidance. Items (e.g., "In order to avoid feelings of disappointment, I just try not to get too serious about work/school" and "I tend to make up excuses to get out of social activities") are rated on a five-point scale $(1=$ "Not at all true for me" to $5=$ "Extremely true for me"). 
2.2.1.9 Experiential avoidance was measured using the 7-item Acceptance and Action Questionnaire (AAQ-II; Bond et al., 2015; $\alpha=.94, .93$ ). Higher scores indicate greater experiential avoidance, which represents difficulty in accepting painful emotional experiences (Hayes, Luoma, Bond, Masuda, \& Lillis, 2006). Experiential avoidance has been conceptualized as a transdiagnostic process associated with the development of anxiety and depression (Levin et al., 2014).

\subsubsection{Post-Pandemic Only}

2.2.2.1 COVID-19 Disruption. Participants were asked the extent that COVID-19 and related impacts have disrupted their daily activities ( $1=$ "None at all," to $5=$ "A great deal"). Participants indicated the degree to which COVID-19 had a negative impact on their health, other people in their lives, their finances, their education, employment and relationships (1 'Strongly disagree", 5 = "Strongly agree"). Participants indicated the extent to which they are worried about future consequences of COVID-19 across these same six domains.

2.2.2.2 COVID-19 Protective Behavior Changes. Participants indicated the extent to which they engaged in protective behaviors including increasing handwashing, increasing hand sanitizer use, avoiding close contact with people who are sick, avoiding close contact with people who are not sick, avoiding spending time with people outside the home, covering their faces when coughing or sneezing, and cleaning/disinfecting surfaces $(1=$ "None at all", $5=$ "A great deal."

2.2.2.3 Housing. Participants were asked about their living circumstances (e.g., with family, roommates, alone, or other) and the number of individuals currently living in the household. 
2.2.2.4 Online Social Engagement. The scale of in-person engagement (Kim et al., 2016) was modified to include the 5 items assessing virtual social engagement (e.g., "The following questions are about how often you have engaged in social activities VIRTUALLY OR ONLINE in the past week"). Participants were asked, "How often have you... used videochat (Zoom, Skype, Facetime) to attend gatherings with friends or family", "Used videochat (Zoom, Skype, Facetime) to attend organized events (e.g., school, work, other)", "Texted friends or family", "Called friends or family" and, "Used social media to interact with friends or family." Participants responded using a 5-point scale ( $1=$ "Very rarely" to $5=$ "Very frequently"). The scale did not have adequate reliability $(\alpha=.66)$ with the inclusion of the two videochat items. As such, these items were excluded, resulting in adequate reliability $(\alpha=.74)$.

2.2.2.5 Problematic internet use was assessed using the Problematic Internet Use Questionnaire-Short Form (PIUQ-SF-6; Demetrovics et al., 2016; $\alpha=.81$ ). This 6-item scale assesses problematic internet use amongst participants, specifically pertaining to how their internet habits cause negative impacts on their daily lives and wellbeing. Items (e.g., How often do you spend time online when you'd rather sleep?") are rated on a 5-point scale (1 = "never" to $5=$ "always/almost always").

2.2.2.6 Perceived benefits related to adversity were assessed using the General Benefit Finding Scale (Cassidy, McLaughlin, \& Giles, 2014; $\alpha=.96$ ). Participants were asked to indicate on a 5-point Likert scale how much each of the 27 items were true for them. These items assess the ability of participants to find perceived benefits in the face of adversity (e.g., "The following questions are about how you have responded to the spread of COVID-19 and related disruptions to your life. In the last month, the spread of COVID-19 and related disruptions to my life... Led me to be more accepting of things."). 
2.2.2.7 Stress was assessed using the vulnerability subscale of the Perceived Stress Scale (PSS; Wickrama et al., 2013; $\alpha=.87$ ). This measure consists of 7 items assessing the psychological vulnerability of participants (e.g., How often have you felt difficulties were piling up so high that you could not overcome them?"). Participants rated each item on a 5-point scale of ( 0 "never" to $4=$ "very often"). Higher scores represent greater psychological vulnerability

\section{Results}

\subsection{COVID-19 Disruption and Protective Behavior Changes.}

More than half $(55.8 \%)$ of participants reported a great deal of disruption to daily activities, with $28 . \%$ reporting "a lot," of disruption, $12.6 \%$ reporting "a moderate amount," $12.6 \%$ reporting "a little," and $.5 \%$ reporting none at all. Education was the area reported by the largest number as significantly impacted, with $93.3 \%$ reported a significant negative impact on their education (See Table 1). Nearly all participants (94.7\%) reported worry about the effect of COVID-19 on others in their lives and on their education $(89.8 \%)$.

Participants reported engaging in protective behavior, with the majority of participants reporting at least a moderate amount of change, such as increasing handwashing (91.8\%), and avoiding close contact with people who are sick (96.1\%). Results are presented in Table 1.

Independent samples t-tests were conducted to examine differences in COVID-19-related disruption at T4. Women reported greater disruption to daily activities $(M=4.45, S D=.75)$ than did their male counterparts $(M=4.13, \mathrm{SD}=1.04), t(202)=-2.32, p=.022)$. Women also reported greater negative impact on their own health $(M=3.30, S D=1.17)$ than $\operatorname{did}$ men $(M=$ $2.74, \mathrm{SD}=1.29) t(202)=-2.78, p=.006$, as well as a more negative financial impact $(M=3.93$, $S D=1.10)$ than did men $(M=3.53, \mathrm{SD}=1.10), t(202)=-2.08, p=.039$. Women also reported more worry about their own health $(M=4.04, S D=1.09)$ than men $(M=3.47, S D=1.25)$. A 
one-way ANOVA was conducted to examine differences in race/ethnicity in on COVID-19related disruption and worry. No differences were found (all $p s>.05$ ).

\subsection{Changes in Anxiety and Depression Symptom Severity Following COVID-19}

A repeated measures ANOVA was conducted to examine depression and anxiety symptom severity post-COVID-19 to symptom severity 2,5 , and 8 months prior. A significant effect of time was found for depression symptom severity, $F(3,167)=22.90, p<.001, \eta_{p}^{2}=.29$. Pairwise comparisons suggested that post-COVID-19 levels of depression was the only time that differed significantly $(p<.05)$ from any other time (Table 2$)$. The mean PHQ-9 score in April (T4) was $12.09(\mathrm{SE}=.54,95 \% \mathrm{CI}[11.03,13.16])$, greater than $\mathrm{T} 1(M=8.91, \mathrm{SE}=.46,95 \% \mathrm{CI}$ $[7.99,9.82]), \mathrm{T} 2(M=9.55, \mathrm{SE}=.46,95 \% \mathrm{CI}[8.64,10.45])$, and T3 $(M=8.61, \mathrm{SE}=.48,95 \%$ CI [7.66, 9.55]). Results are presented in Figure 1.

A significant effect of time was found for anxiety symptom severity, $F(3,167)=13.00, p$ $<.001, \eta_{p}{ }^{2}=.19$. Pairwise comparisons indicated that T1 did not differ from T2 $(p=.143) . \mathrm{T} 3$ (February) differed from T1, $\left(M_{\text {Diff }}=-.97, p=.016\right)$ with participants reporting a mean of 8.29 $(95 \% \mathrm{CI}[7.43,9.15])$ at the beginning of the school year and $7.32(95 \% \mathrm{CI}[6.38,8.27])$ at 6 months into the academic year. Results are presented in Figure 2. Post-COVID-19 levels of anxiety $(\mathrm{M}=9.71,95 \% \mathrm{CI}[8.77,10.64]$ were significantly higher than all other time points in the year (Table 2). ${ }^{2}$

\footnotetext{
${ }^{2}$ The sample represents a subset of participants (55\% of enrolled sample) randomized to receive a single-session, 30-minute, online intervention including growth mindset and information about modifiable risk factors for depression and anxiety. Among this group, a between-subjects, repeated measures ANOVA indicated no significant interaction between Condition and anxiety symptom severity, $F(3,166)=.812, p=.489$ or depression symptom severity $\mathrm{F}(3,166)=.25, p=.862$. GAD-7 scores and PHQ-9 scores over time by condition are presented in the supplementary materials. An ANCOVA, however, indicated that when controlling for baseline emotion malleability beliefs, the condition had a statistically significant effect on beliefs that emotions were malleable at $\mathrm{T} 4, \mathrm{~F}(1,198)=$ $.52, p=.025$. Participants in the intervention group endorsed a greater belief in the malleability of emotions $(\mathrm{M}=$ $3.74, \mathrm{SE}=.08,95 \% \mathrm{CI}[3.59,3.89])$ than did their counterparts in the intervention group $(\mathrm{M}=3.49, \mathrm{SE}=.08,95 \%$ CI $[3.34,3.65])$. As such, the intervention did not have direct effects on depression and anxiety symptom severity at any given time point, yet may still have had an effect on variables relevant to the present analysis.
} 
It should be noted that these increases in depression and anxiety symptoms were associated with a drastic increase in those that exceeded clinical cutoffs indicating pathology using the PHQ-9 for depression and the GAD-7 for anxiety. In February (T3), 128, or 62.1\% of the sample were below the clinical cutoff score of 10 on the PHQ-9. Of this group, 53 (41.4\%) were above this threshold at following COVID-19. Of the 137 (66.5\%) participants below GAD7 cutoffs in February, 50 (36.5\%) were above the cutoff in April.

\subsection{Predicting Psychological Distress}

Multiple linear regression analysis was used to examine predictors of depression symptom severity, anxiety symptom severity, and perceived stress during the COVID-19 pandemic, controlling for psychological distress prior to the impact of COVID-19. Predictors included demographic variables (i.e. gender, race, income), living circumstances (with family, number of individuals in the home), health behaviors (social contact, online social contact, internet use, moderate physical activity, diet, and sleep quality (i.e. hours slept per night). The model also included psychological variables including perceived malleability of emotion, cognitive and behavioral avoidance, and experiential avoidance.

When controlling for pre-existing depression and anxiety symptom severity, female gender was associated with increases in perceived stress $(\beta=0.14, p=.030)$ and approaching significance for greater anxiety symptom severity $(\beta=0.12, p=.053)$. Relative to their White counterparts, Asian or Asian-American-identifying participants reported lower levels of depression $(\beta=-.15, p=.011)$ and anxiety $(\beta=-0.15, p=.013)$. Participants identifying as Latinx reported greater depression symptom severity $(\beta=0.12, p=.044)$.

For health behaviors, greater online social contact (texting, phone calls, and social media use) was significantly associated with higher PHQ-9 scores $(\beta=0.12, p=.027)$. Increases in 
problematic internet use was associated with increases in anxiety symptom severity $(\beta=0.18, p$ $=.005)$ and approaching significance for greater depression symptom severity $(\beta=0.12, p=$ $.055)$.

In examining the psychological factors that might predict psychological well-being, cognitive and behavioral avoidance was associated with more distress across all outcomes. Increased avoidance was associated with greater depression symptom severity $(\beta=0.28, p=$ $.001)$, anxiety symptom severity $(\beta=0.21, p=.015)$, and perceived stress $(\beta=0.27, p=.003)$. Increases in experiential avoidance approached significance for being associated with greater stress $\beta=0.19, p=.058$. Increases in perceived benefits as a result of COVID-19 approached significance for being associated with lower depression symptom severity $(\beta=-0.11, p=.058)$. Results are presented in Table 3.

\subsection{Predicting Post-COVID-19 Distress from Pre-COVID-19 Factors}

The same health behaviors and psychological variables reported prior to the COVID-19 spread (T3) and related impact were then examined as predictors of psychological distress during the pandemic. Controlling for demographic variables, only pre-existing symptom severity at T3 were related to symptom severity during the outbreak (T4), with T3 depression severity predicting levels of depression symptoms at T4 $(\beta=.54, p<.001)$, and only T3 anxiety symptom severity $(\beta=.39, p<.001)$ and T3 depression severity approaching significance $(\beta=$ $.19, p=.053$ ) associated with T4 anxiety levels. For perceived stress, cognitive and behavioral avoidance at T3 was a significant predictor of stress at T4, with greater avoidance associated with more stress $(\beta=.25, p=.008)$. Results are presented in Table 4.

\section{Discussion}


The present study examined the course of anxiety and depression symptom severity among college students during the 2019-2020 academic year, before and after the COVID-19 pandemic and related impacts. Findings suggested a drastic increase in psychological distress in this population from February to April of 2020. Mean PHQ-9 and GAD-7 scores increased by 4.3 and 3.84 points, respectively. Mean PHQ-9 scores were above the clinically significant range for the whole sample. These findings are consistent with past studies showing high distress among students during the COVID-19 pandemic (Wang et al., 2020). Further, greater length of quarantine has been found to be associated with more distress (Brooks et al., 2020). The present study adds to extant research on the mental health effects of the COVID-19 pandemic by demonstrating the magnitude of this change relative to mental health throughout the academic year.

Factors predicting psychological distress during the COVID-19 pandemic when controlling for distress two months prior were examined. In keeping with prior findings, women were more likely to experience greater anxiety symptom severity and greater stress (Moccia et al., 2020; Wang et al., 2020). This finding is particularly notable, as analyses controlled for mental health symptom severity before the pandemic, suggesting an outsized impact on female college students. This result may be explained by female students' greater self-report of disruption and impact on their health and financial circumstances. Latinx participants also reported higher depression symptom severity compared with their white counterparts, whereas Asian and Asian-American participants reported lower mental health symptom severity. No differences in between groups, however, were found in self-reported impact of the pandemic.

When controlling for pre-existing distress, online social contact and problematic internet use were associated with greater distress. These findings are consistent with a relationship 
between problematic internet use and greater depression symptom severity (Demetrovics et al., 2016). These findings could reflect a relationship between pandemic-related media exposure and distress. For instance, in response to the Ebola crises, media prior to the crises, greater media exposure to Ebola-related news, and prior mental health diagnoses were all associated with greater Ebola-related worry, distress and impairment (Thompson, Garfin, Holman, \& Silver, 2017). It is also possible that students engaging in more online social contact had a greater need for accessing social support, perhaps suggesting a greater disruption imposed by social distancing practices.

The most consistent predictor of distress was cognitive and behavioral avoidance, which predicted depression, anxiety and distress during the pandemic. This finding is consistent with research suggesting avoidance increases risk for both depression and anxiety (Hofmann, 2007; Ottenbreit \& Dobson, 2008). Of course, avoidance of exposure to the coronavirus is part of the public health measures necessary to address the pandemic. However, items on the Cognitive and Behavioral Avoidance scale reflect avoidance of both safe situations and also avoidance of difficult thoughts (Ottenbreit \& Dobson, 2004). Furthermore, avoidance prior to the pandemic also predicted greater stress during the pandemic, suggesting that the tendency to avoid difficult situations conferred risk even prior to mandatory social distancing.

Other health behaviors that have been shown to increase risk for depression and anxiety such as poor sleep quality, low physical activity, low social engagement, and unhealthy diet (Cairns et al., 2014; Emerson et al., 2018; Zimmermann, Chong, Vechiu, \& Papa, 2020) were not associated with greater distress. Similarly, while believing emotions to be malleable has been shown to predict distress in college students across the college transition (Kneeland \& Dovidio, 2019; Levin et al., 2012; Tamir, John, Srivastava, \& Gross, 2007), endorsement of this belief as 
not associated with distress during the pandemic. It may be that these factors did not serve as protective factors immediately following the onset of the COVID-19 pandemic but could confer benefit in the long-term.

Because mental health during the COVID-19 pandemic was only assessed at one time point, it is unclear if these results will persist for any meaningful length of time. Notably, a 4 to 6 month follow-up of individuals quarantine during the spread of the Middle East Respiratory Syndrome (MERS) found elevated anxiety during isolation that decreased at follow-up. Past psychiatric history and medical history, medical costs, financial losses and lack of sufficient food, clothing and supplies, however, were associated with persistence of anxiety following isolation (Jeong et al., 2016). As such, attending to these psychosocial stressors may be vital to preventing the persistence of anxiety symptoms as shelter-in-place measures remain. The expansion of telehealth services and self-guided internet intervention services will also be essential to the alleviation of this distress in the long term (Fischer et al., 2020; Gruber et al., 2020).

\subsection{Limitations}

This study has several important limitations. The primary purpose of the study was to examine the effect of an intervention on depression and anxiety in August 2019. While the intervention did not appear to have an impact on depression or anxiety at T4, it did influence the intended construct of perceived malleability of emotion at this time. As such, it is possible that students not given this intervention may have responded differently. Given the observational nature of the study, it is not possible to draw causal conclusions regarding the effect of the COVID-19 pandemic. A strength of this study, however, was the ability to examine predictors 
when controlling for pre-existing mental health, thereby strengthening the causal direction of the interpretation.

The study was also limited by the potential for self-selection bias. The sample included only those participants who responded to a follow-up eight months following study enrollment. The sample was disproportionately female (76.2\%), which is also generally true of college students on the whole (65.0\% women; ACHA, 2018), although may be a larger share in the present study. These aspects may limit the generalizability of the results. Furthermore, the generalizability to other populations beyond college students is unclear. College students likely face consequences of the pandemic, such as moving home after living independently, that may differ from the general population.

\section{2 Conclusion}

The present study examined college student mental health over the course of the 20192020 academic year, prior to and during the COVID-19 pandemic. Psychological research suggests that the multifaceted stressors accompanying the COVID-19 pandemic may be particularly likely to negatively impact mental health at a large scale due to the high degree of uncertainty, disruption to daily life, and economic impact (Gruber et al., 2020). These predictions were supported, as results suggested a striking increase in depression and anxiety among a college student sample. Certain groups may be at elevated risk for mental health concerns, including individuals with pre-existing psychological distress, women, and Latinx-identifying students. The present study also identified behaviors associated with greater distress during the pandemic, including online social contact, problematic internet use, and cognitive and behavioral avoidance. These factors may be important targets for intervention. Only pre-existing depression 
and anxiety and avoidance behavior prior to the pandemic was associated with distress during the pandemic. 


\section{References}

ACHA. (2018). American College Health Association-National College Health Assessment II: University of Nevada Reno Executive Summary. Hanover, MD.

Bond, F. W., Hayes, S. C., Carpenter, K. M., Orcutt, H. K., Waltz, T., Baer, R. A., ... Waltz, T. (2015). Preliminary psychometric properties of the Acceptance and Action Questionnaire II : A revised measure of psychological flexibility and Preliminary Psychometric Properties of the Acceptance and Action Questionnaire - II : A Revised Measure of Psychologic. Behavior Therapy, 42(4), 676-688. https://doi.org/10.1016/j.beth.2011.03.007

Brooks, S. K., Webster, R. K., Smith, L. E., Woodland, L., Wessely, S., Greenberg, N., \& Rubin, G. J. (2020). The psychological impact of quarantine and how to reduce it: rapid review of the evidence. The Lancet.

Cairns, K. E., Yap, M. B. H., Pilkington, P. D., \& Jorm, A. F. (2014). Risk and protective factors for depression that adolescents can modify: a systematic review and meta-analysis of longitudinal studies. Journal of Affective Disorders, 169, 61-75. https://doi.org/10.1016/j.jad.2014.08.006

Cao, W., Fang, Z., Hou, G., Han, M., Xu, X., Dong, J., \& Zheng, J. (2020). The psychological impact of the COVID-19 epidemic on college students in China. Psychiatry Research, 112934.

Cassidy, T., McLaughlin, M., \& Giles, M. (2014). Benefit finding in response to general life stress: measurement and correlates. Health Psychology and Behavioral Medicine: An Open Access Journal, 2(1), 268-282.

Demetrovics, Z., Király, O., Koronczai, B., Griffiths, M. D., Nagygyörgy, K., Elekes, Z., ... Urban, R. (2016). Psychometric properties of the Problematic Internet Use Questionnaire 
Short-Form (PIUQ-SF-6) in a nationally representative sample of adolescents. PloS One, 11(8).

Dong, E., Du, H., \& Gardner, L. (2020). An interactive web-based dashboard to track COVID-19 in real time. The Lancet Infectious Diseases.

Emerson, N. D., Small, G. W., Merrill, D. A., Chen, S. T., Torres-Gil, F., \& Siddarth, P. (2018). Behavioral risk factors for self-reported depression across the lifespan. Mental Health \& Prevention, 12, 36-41. https://doi.org/https://doi.org/10.1016/j.mhp.2018.09.002

Fischer, R., Karl, J., Bortolini, T., Zilberberg, M., Robinson, K., Rabelo, A. L. A., ... Irving, B. (2020). Rapid review and meta-meta-analysis of self-guided interventions to address anxiety, depression and stress during COVID-19 social distancing.

Ford, B. Q., Lwi, S. J., Gentzler, A. L., Hankin, B., \& Mauss, I. B. (2018). The cost of believing emotions are uncontrollable: Youths' beliefs about emotion predict emotion regulation and depressive symptoms. Journal of Experimental Psychology: General, 147(8), 1170-1190. https://doi.org/10.1037/xge0000396

Gruber, J., Prinstein, M., Abramowitz, J. S., Albano, A. M., Aldao, A., Borelli, J., \& ...Weinstock, L. (2020). Clinical Psychological Science's Call To Action in the Time of COVID-19. https://doi.org/https://doi.org/10.31234/osf.io/desg9

Hayes, S. C., Luoma, J. B., Bond, F. W., Masuda, A., \& Lillis, J. (2006). Acceptance and Commitment Therapy: Model, processes and outcomes. Behaviour Research and Therapy, 44(1), 1-25. https://doi.org/10.1016/J.BRAT.2005.06.006

Hays, R. D., Martin, S. A., Sesti, A. M., \& Spritzer, K. L. (2005). Psychometric properties of the medical outcomes study sleep measure. Sleep Medicine, 6(1), 41-44. https://doi.org/https://doi.org/10.1016/j.sleep.2004.07.006 
Hofmann, S. G. (2007). Cognitive factors that maintain social anxiety disorder: A comprehensive model and its treatment implications. Cognitive Behaviour Therapy, 36(4), 193-209. https://doi.org/https://doi.org/10.1080/16506070701421313

Jeong, H., Yim, H. W., Song, Y.-J., Ki, M., Min, J.-A., Cho, J., \& Chae, J.-H. (2016). Mental health status of people isolated due to Middle East Respiratory Syndrome. Epidemiology and Health, 38 .

Kim, Y., Wang, Y., \& Oh, J. (2016). Digital media use and social engagement: How social media and smartphone use influence social activities of college students. Cyberpsychology, Behavior, and Social Networking, 19(4), 264-269. https://doi.org/https://doi.org/10.1089/cyber.2015.0408

Kneeland, E. T., \& Dovidio, J. F. (2019). Emotion malleability beliefs and coping with the college transition. Emotion. https://doi.org/10.1037/emo0000559

Kroenke, K., Spitzer, R. L., \& Williams, J. B. W. (2001). The PHQ-9: validity of a brief depression severity measure. Journal of General Internal Medicine, 16(9), 606-613. https://doi.org/https://doi.org/10.1046/j.1525-1497.2001.016009606.x

Levin, M. E., Lillis, J., Seeley, J., Hayes, S. C., Pistorello, J., \& Biglan, A. (2012). Exploring the relationship between experiential avoidance, alcohol use disorders, and alcohol-related problems among first-year college students. Journal of American College Health, 60(6), $443-448$.

Levin, M. E., MacLane, C., Daflos, S., Seeley, J. R., Hayes, S. C., Biglan, A., \& Pistorello, J. (2014). Examining psychological inflexibility as a transdiagnostic process across psychological disorders. Journal of Contextual Behavioral Science, 3(3), 155-163.

Marshall, A. L., Smith, B. J., Bauman, A. E., \& Kaur, S. (2005). Reliability and validity of a 
brief physical activity assessment for use by family doctors. British Journal of Sports Medicine, 39(5), 294-297.

Moccia, L., Janiri, D., Pepe, M., Dattoli, L., Molinaro, M., De Martin, V., ... Sani, G. (2020). Affective temperament, attachment style, and the psychological impact of the COVID-19 outbreak: an early report on the Italian general population. Brain, Behavior, and Immunity.

Ottenbreit, N. D., \& Dobson, K. S. (2004). Avoidance and depression: the construction of the Cognitive-Behavioral Avoidance Scale. Behaviour Research and Therapy, 42(3), 293-313. https://doi.org/https://doi.org/10.1016/S0005-7967(03)00140-2

Ottenbreit, N. D., \& Dobson, K. S. (2008). Avoidance. In Risk factors in depression (pp. 447470). Elsevier. https://doi.org/https://doi.org/10.1016/B978-0-08-045078-0.00020-4

Schroder, H. S., Dawood, S., Yalch, M. M., Donnellan, M. B., \& Moser, J. S. (2016). Evaluating the Domain Specificity of Mental Health-Related Mind-Sets. Social Psychological and Personality Science, 7(6), 508-520. https://doi.org/10.1177/1948550616644657

Spitzer, R. L., Kroenke, K., Williams, J. B. W., \& Löwe, B. (2006). A brief measure for assessing generalized anxiety disorder: the GAD-7. Archives of Internal Medicine, 166(10), 1092-1097. https://doi.org/doi:10.1001/archinte.166.10.1092

Tamir, M. (2007). Implicit Theories of Emotion: Affective and Social Outcomes Across a Major Life Transition. Article in Journal of Personality and Social Psychology. https://doi.org/10.1037/0022-3514.92.4.731

Tamir, M., John, O. P., Srivastava, S., \& Gross, J. J. (2007). Implicit theories of emotion: affective and social outcomes across a major life transition. Journal of Personality and Social Psychology, 92(4), 731-744. https://doi.org/10.1037/0022-3514.92.4.731

Thompson, R. R., Garfin, D. R., Holman, E. A., \& Silver, R. C. (2017). Distress, worry, and 
functioning following a global health crisis: A national study of Americans' responses to Ebola. Clinical Psychological Science, 5(3), 513-521.

Wang, C., Pan, R., Wan, X., Tan, Y., Xu, L., Ho, C. S., \& Ho, R. C. (2020). Immediate psychological responses and associated factors during the initial stage of the 2019 coronavirus disease (COVID-19) epidemic among the general population in China. International Journal of Environmental Research and Public Health, 17(5), 1729.

Wickrama, K. A. S., Ralston, P. A., O’Neal, C. W., Ilich, J. Z., Harris, C. M., Coccia, C., ... Lemacks, J. (2013). Linking life dissatisfaction to health behaviors of older African Americans through psychological competency and vulnerability. Research on Aging, 35(5), $591-611$.

Zazpe, I., Bes-Rastrollo, M., Ruiz-Canela, M., Sánchez-Villegas, A., Serrano-Martínez, M., \& Martínez-González, M. A. (2011). A brief assessment of eating habits and weight gain in a Mediterranean cohort. British Journal of Nutrition, 105(5), 765-775.

Zhou, F., Yu, T., Du, R., Fan, G., Liu, Y., Liu, Z., ... Gu, X. (2020). Clinical course and risk factors for mortality of adult inpatients with COVID-19 in Wuhan, China: a retrospective cohort study. The Lancet.

Zimmermann, M., Chong, A. K., Vechiu, C., \& Papa, A. (2020). Modifiable risk and protective factors for anxiety disorders among adults: A systematic review. Psychiatry Research, 285, 112705. https://doi.org/https://doi.org/10.1016/j.psychres.2019.112705 


\section{Figure 1}

Depression Symptom Severity During 2019-2020 Academic Year

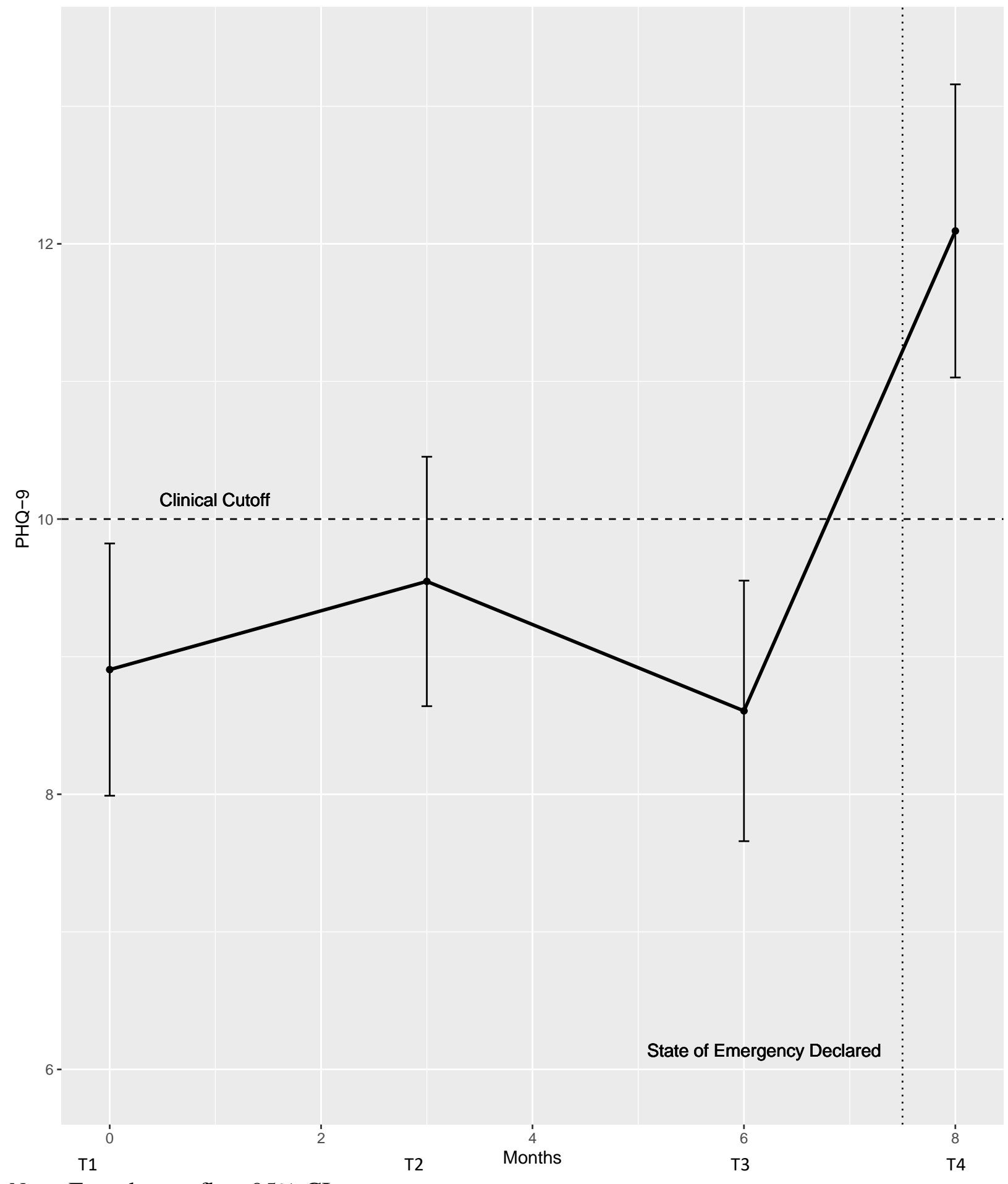

Note: Error bars reflect $95 \% \mathrm{CI}$. 


\section{Figure 2}

Anxiety Symptom Severity During 2019-2020 Academic Year

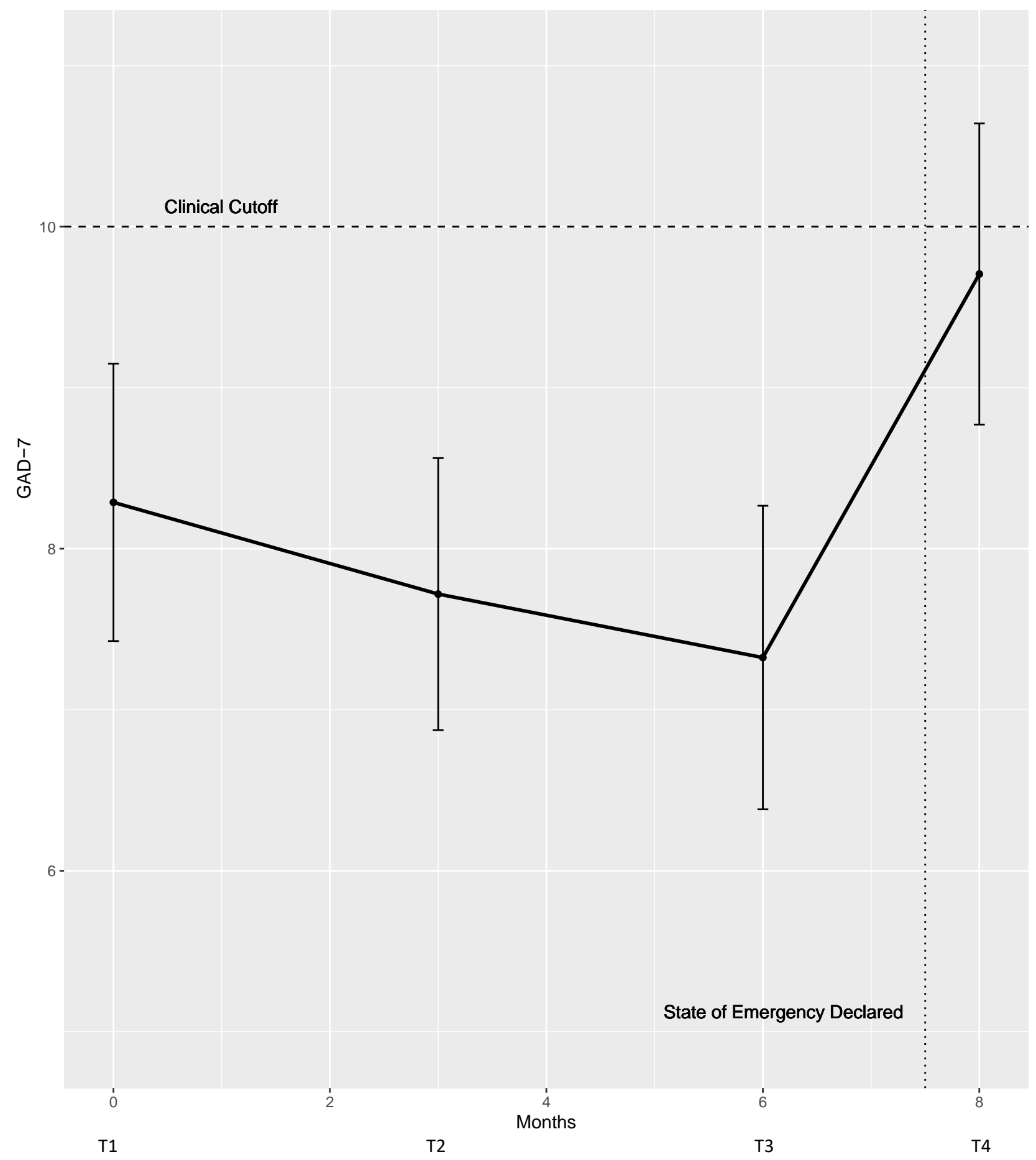

Note: Error bars reflect $95 \% \mathrm{CI}$. 
Table 1

Self-reported Impact of COVID-19

\begin{tabular}{|c|c|c|c|c|c|}
\hline & $\begin{array}{l}\text { Strongly } \\
\text { disagree } \\
(\%)\end{array}$ & $\begin{array}{l}\text { Somewhat } \\
\text { disagree } \\
(\%)\end{array}$ & $\begin{array}{l}\text { Neither agree } \\
\text { nor disagree } \\
(\%)\end{array}$ & $\begin{array}{l}\text { Somewhat } \\
\text { agree }(\%)\end{array}$ & $\begin{array}{l}\text { Strongly } \\
\text { agree }(\%)\end{array}$ \\
\hline \multicolumn{6}{|l|}{$\begin{array}{l}\text { The COVID- } 19 \text { spread has already had a } \\
\text { significant negative effect on... }\end{array}$} \\
\hline My health & 14.6 & 14.1 & 18.9 & 43.7 & 8.7 \\
\hline Others I know & 3.4 & 4.9 & 9.8 & 42 & 40 \\
\hline My financial circumstances & 5.8 & 8.7 & 14.6 & 37.4 & 33.5 \\
\hline My education & 2.4 & 1.5 & 2.9 & 25.7 & 67.5 \\
\hline My employment & 14.1 & 6.3 & 25.2 & 16 & 38.3 \\
\hline \multicolumn{6}{|l|}{$\begin{array}{l}\text { I am worried about the effect of COVID- } \\
19 \text { on... }\end{array}$} \\
\hline My health & 5.8 & 9.7 & 6.3 & 42.7 & 35.4 \\
\hline Others I know & 0 & 4.4 & 1 & 24.8 & 69.9 \\
\hline My financial circumstances & 4.9 & 5.3 & 11.7 & 25.7 & 52.4 \\
\hline My education & 1.5 & 3.4 & 5.3 & 18.9 & 70.9 \\
\hline My employment & 6.8 & 8.7 & 16.5 & 18.9 & 49 \\
\hline My relationships & 3.9 & 8.3 & 11.2 & 33 & 43.7 \\
\hline Behavior Change in Past Month & $\begin{array}{l}\text { None at all } \\
(\%)\end{array}$ & A little $(\%)$ & $\begin{array}{l}\text { A moderate } \\
\text { amount }(\%)\end{array}$ & $\mathrm{A} \operatorname{lot}(\%)$ & $\begin{array}{l}\text { A great } \\
\text { deal }(\%)\end{array}$ \\
\hline Increased handwashing & 1.5 & 6.8 & 16.5 & 31.1 & 44.2 \\
\hline $\begin{array}{l}\text { Increased hand sanitizer use } \\
\text { Avoiding close contact with people }\end{array}$ & 11.2 & 14.1 & 21.8 & 25.7 & 27.2 \\
\hline who are sick & 1 & 2.9 & 9.2 & 26.7 & 60.2 \\
\hline $\begin{array}{l}\text { Avoiding close contact with people } \\
\text { who are healthy }\end{array}$ & 5.4 & 11.7 & 26.8 & 30.7 & 25.4 \\
\hline $\begin{array}{l}\text { Avoiding spending time with people } \\
\text { outside of my home }\end{array}$ & 1.5 & 8.3 & 9.7 & 22.3 & 58.3 \\
\hline Cover my face when I cough or sneeze & 9.2 & 7.3 & 13.1 & 22.3 & 48.1 \\
\hline Cleaning/disinfecting surfaces & 1.5 & 14.6 & 18.9 & 21.4 & 43.7 \\
\hline
\end{tabular}




\section{Table 2}

Pairwise Comparisons of Mean Depression and Anxiety Symptom Severity During 2019-2020 Academic Year

\begin{tabular}{cccccc}
\hline & & \multicolumn{3}{c}{ 95 \% CIDiff } \\
& M Diff & SE $_{\text {Diff }}$ & $p$ & Lower & Upper \\
\hline PHQ-9 & & & & & \\
T4 vs. T1 & 3.19 & 0.51 & $<.000$ & 2.18 & 4.20 \\
T4 vs. T2 & 2.55 & 0.49 & $<.000$ & 1.59 & 3.50 \\
T4 vs. T3 & 3.49 & 0.43 & $<.000$ & 2.64 & 4.34 \\
GAD-7 & & & & & \\
T4 vs. T1 & 1.42 & 0.41 & .001 & 0.61 & 2.23 \\
T4 vs. T2 & 1.99 & 0.40 & $<.000$ & 1.19 & 2.78 \\
T4 vs. T3 & 2.38 & 0.40 & $<.000$ & 1.59 & 3.18 \\
\hline
\end{tabular}

Note. T1: August 2019, T2: December 2019, T3: February 2020, T4: April 2020 
Table 3

Predictors of Psychological Distress during COVID-19 Controlling for Pre-COVID-19 Distress

\begin{tabular}{|c|c|c|c|c|c|c|c|c|c|}
\hline & \multicolumn{9}{|c|}{ Psychological Distress During the COVID-19 Pandemic (April 2020 [T4]) } \\
\hline & \multicolumn{3}{|c|}{ Depression (PHQ-9) } & \multicolumn{3}{|c|}{ Anxiety (GAD-7) } & \multicolumn{3}{|c|}{ Stress (PSS) } \\
\hline & $b$ & $\beta$ & $p$ & $b$ & $\beta$ & $p$ & $b$ & $\beta$ & $p$ \\
\hline \multicolumn{10}{|l|}{ Demographics } \\
\hline Female (vs. Male) & 0.46 & 0.03 & .620 & 1.62 & 0.12 & .053 & 0.27 & 0.14 & .030 \\
\hline Latinx (vs. White) & 2.22 & 0.12 & .044 & -0.59 & -0.04 & .550 & -0.09 & -0.04 & .540 \\
\hline Black (vs. White) & -1.32 & -0.03 & .528 & -0.94 & -0.03 & .618 & 0.29 & 0.06 & .316 \\
\hline Asian (vs. White) & -2.60 & -0.15 & .011 & -2.27 & -0.15 & .013 & -0.04 & -0.02 & .779 \\
\hline Other (vs. White) & -1.08 & -0.04 & .451 & -0.46 & -0.02 & .725 & -0.05 & -0.02 & .797 \\
\hline Income Middle (vs. low) & -1.01 & -0.07 & .265 & 1.16 & 0.09 & .155 & -0.12 & -0.07 & .317 \\
\hline Income High (vs. low) & -1.14 & -0.08 & .211 & 0.64 & 0.05 & .436 & 0.14 & 0.08 & .269 \\
\hline \multicolumn{10}{|l|}{ T4 Housing Circumstances } \\
\hline Living with family & 0.12 & 0.01 & .905 & -0.38 & -0.02 & .680 & -0.20 & -0.09 & .156 \\
\hline Household size & 0.13 & 0.03 & .636 & -0.01 & 0.00 & .971 & 0.06 & 0.11 & .118 \\
\hline \multicolumn{10}{|l|}{ T4 Health Behaviors } \\
\hline Healthy Diet & 0.29 & 0.10 & .085 & 0.03 & 0.01 & .848 & -0.01 & -0.04 & .523 \\
\hline PA 1-2x/week (vs. none) & -0.07 & -0.01 & .954 & 0.39 & 0.03 & .715 & -0.05 & -0.03 & .763 \\
\hline PA 3-4x/week (vs. none) & 1.83 & 0.13 & .115 & 0.17 & 0.01 & .869 & 0.20 & 0.12 & .195 \\
\hline PA $>5 x /$ week (vs. none) & 0.05 & 0.00 & .971 & 0.28 & 0.02 & .806 & 0.27 & 0.14 & .121 \\
\hline Hours of Sleep & -0.03 & -0.08 & .143 & -0.02 & -0.07 & .217 & 0.00 & 0.02 & .790 \\
\hline Social contact & -0.16 & -0.03 & .628 & -0.06 & -0.01 & .843 & 0.02 & 0.02 & .739 \\
\hline Online social contact & 1.00 & 0.12 & .027 & 0.58 & 0.08 & .152 & 0.10 & 0.10 & .111 \\
\hline Problematic Internet Use & 0.97 & 0.12 & .055 & 1.30 & 0.18 & .005 & 0.09 & 0.09 & .200 \\
\hline \multicolumn{10}{|l|}{ T4 Psychological Variables } \\
\hline Cog Beh Avoidance & 0.08 & 0.28 & .001 & 0.05 & 0.21 & .015 & 0.01 & 0.28 & .003 \\
\hline Experiential Avoidance & 0.04 & 0.05 & .542 & 0.02 & 0.04 & .677 & 0.02 & 0.19 & .058 \\
\hline \multicolumn{10}{|l|}{ Emotion Malleability } \\
\hline Beliefs & -0.18 & -0.03 & .653 & 0.04 & 0.01 & .913 & -0.07 & -0.08 & .221 \\
\hline \multirow[t]{2}{*}{ Perceived Benefits } & -0.88 & -0.11 & .058 & -0.36 & -0.05 & .394 & -0.01 & -0.02 & .817 \\
\hline & \multicolumn{3}{|c|}{$R^{2}=.60, A R^{2}=.54$} & \multicolumn{3}{|c|}{$R^{2}=.55, A R^{2}=.49$} & \multicolumn{3}{|c|}{$R^{2}=.46, A R^{2}=.38$} \\
\hline
\end{tabular}

Note: Analyses control for pre-COVID-19 mental health (PHQ-9 and GAD-7 in February 2020

[T3]). All models were significant, $p<.001$. 


\section{Table 4}

Pre-COVID-19 Predictors of Psychological Distress During COVID-19

\begin{tabular}{|c|c|c|c|c|c|c|c|c|c|}
\hline & \multicolumn{9}{|c|}{ Psychological Distress During the COVID-19 Pandemic (April 2020 [T4]) } \\
\hline & \multicolumn{3}{|c|}{ Depression (PHQ-9) } & \multicolumn{3}{|c|}{ Anxiety (GAD-7) } & \multicolumn{3}{|c|}{ Stress (PSS) } \\
\hline & $b$ & $\beta$ & $p$ & $b$ & $\beta$ & $p$ & $b$ & $\beta$ & $p$ \\
\hline \multicolumn{10}{|l|}{ T3 Psychological Distress } \\
\hline PHQ-9 & 0.63 & 0.55 & .000 & 0.19 & 0.19 & .053 & 0.01 & 0.08 & .486 \\
\hline GAD-7 & 0.01 & 0.01 & .952 & 0.45 & 0.44 & .000 & 0.02 & 0.11 & .324 \\
\hline \multicolumn{10}{|l|}{ T3 Health Behaviors } \\
\hline Social contact & 0.22 & 0.03 & .645 & -0.05 & -0.01 & .907 & -0.01 & -0.01 & .840 \\
\hline Hours of Sleep & -0.02 & -0.06 & .263 & -0.01 & -0.03 & .568 & 0.00 & -0.03 & .709 \\
\hline Healthy Diet & 0.04 & 0.02 & .804 & 0.03 & 0.01 & .829 & -0.02 & -0.07 & .354 \\
\hline PA 1-2x/week (vs. none) & -1.00 & -0.07 & .275 & -0.47 & -0.04 & .560 & 0.06 & 0.04 & .650 \\
\hline PA 3-4x/week (vs. none) & -0.59 & -0.03 & .594 & 1.11 & 0.07 & .258 & 0.07 & 0.04 & .637 \\
\hline PA > 5x/week (vs. none) & -2.08 & -0.08 & .182 & -1.74 & -0.08 & .204 & 0.16 & 0.05 & .449 \\
\hline \multicolumn{10}{|l|}{ T3 Psychological Variables } \\
\hline $\begin{array}{l}\text { Cog Beh Avoidance } \\
\text { Emotion Malleability }\end{array}$ & 0.02 & 0.07 & .344 & 0.03 & 0.12 & .118 & 0.01 & 0.25 & .008 \\
\hline Beliefs & -0.30 & -0.04 & .506 & 0.22 & 0.03 & .581 & -0.03 & -0.03 & .673 \\
\hline \multirow[t]{2}{*}{ Experiential Avoidance } & 0.04 & 0.07 & .399 & -0.02 & -0.04 & .693 & 0.01 & 0.09 & .388 \\
\hline & \multicolumn{3}{|c|}{$R^{2}=.52, A R^{2}=.46$} & \multicolumn{3}{|c|}{$R^{2}=.51, A R^{2}=.45$} & \multicolumn{3}{|c|}{$R^{2}=.34, A R^{2}=.26$} \\
\hline
\end{tabular}

Note: Analyses controlled for gender, race, income, and housing. All models were significant, $p$ $<.001$. T3: February 2020, T4: April 2020. 